\title{
Circuit
}

Musiques contemporaines

\section{Festival Elektra 2001}

\section{Réjean Beaucage}

Volume 12, numéro 2, 2002

Opéra aujourd'hui

URI : https://id.erudit.org/iderudit/902255ar

DOI : https://doi.org/10.7202/902255ar

Aller au sommaire du numéro

Éditeur(s)

Les Presses de l'Université de Montréal

ISSN

1183-1693 (imprimé)

1488-9692 (numérique)

Découvrir la revue

Citer ce compte rendu

Beaucage, R. (2002). Compte rendu de [Festival Elektra 2001]. Circuit, 12(2),

81-88. https://doi.org/10.7202/902255ar

Ce document est protégé par la loi sur le droit d'auteur. L'utilisation des services d'Érudit (y compris la reproduction) est assujettie à sa politique d'utilisation que vous pouvez consulter en ligne.

https://apropos.erudit.org/fr/usagers/politique-dutilisation/
Cet article est diffusé et préservé par Érudit.

Érudit est un consortium interuniversitaire sans but lucratif composé de l’Université de Montréal, l'Université Laval et l'Université du Québec à Montréal. Il a pour mission la promotion et la valorisation de la recherche. https://www.erudit.org/fr/ 


\title{
Festival Elektra 2001
}

\author{
Réjean Beaucage
}

Du 8 au 17 novembre 2001 se tenait à Montréal la troisième édition du festival ELEKTRA de l'Association pour la création et la recherche électroacoustiques du Québec (ACREQ), un organisme dont la fondation remonte à 1978 et qui peut prétendre au titre de "premier organisme canadien voué exclusivement au genre électroacoustiquel ». L'importance historique de l'ACREQ dans le développement du genre électroacoustique au Québec est indéniable et nombreux sont les compositeurs et mélomanes qui ont connu leur premier frisson acousmatique dans ses salles obscures. Depuis quelque temps toutefois, il n'est plus aussi fréquent de trouver à l'ACREQ des programmes de musique électroacoustique "institutionnelle», c'est-à-dire cette musique qui vient à la suite de la rencontre sur une table de mixage de la musique concrète de Pierre Schaeffer et de la musique électronique de Karlheinz Stockhausen (je schématise) et dont on enseigne les préceptes dans les conservatoires et universités. Certains voient s'opérer dans les programmes présentés depuis quelques années à l'ACREQ un glissement vers des musiques plus "populaires" au détriment des musiques de recherche. II semble en effet que l'on ait décidé de faire une part sans cesse croissante dans la programmaton à un "nouveau» type d'artistes sonores. Ils sont souvent autodidactes, viennent à la musique après avoir tâter d'autres formes d'art et ne sont pas connu du circuit habituel des concours internationaux de musique électroacoustique, ou ne l'étaient pas jusqu'à récemment. Qui sont donc ces nouveaux créateurs?

On sait évidemment que la lutherie nécessaire à l'élaboration de créations sonores électroniques, d'extrêmement complexe qu'elle était lorsque Stockhausen composait ses premières études en 1953 aux studios de musique électronique de la WDR, est maintenant adaptable à tout ordinateur personnel et peut aisément être utilisée par tout aspirant compositeur. La démocratisation des moyens de production a fait éclater le genre électroacoustique en une myriade de sous-genres que l'on peut regrouper sous l'appellation générale d'arts électroniques, parmi lesquels, évidemment, se trouvent aussi ceux qui relèvent du domaine des arts visuels (vidéo, infographie, etc.). On assiste d'ailleurs depuis quelques années à un phénomène étrange, celui des artistes visuels qui, du jour au lendemain, à la faveur
1. Comme le décrit François Guérin dans son excellent «Aperçu du genre électroacoustique au Québec», publié dans Circuit, vol. 4, $n^{\text {os }} 1-2$, Électroacoustique-Québec: l'essor, PUM, 1993. 
de la découverte d'un programme dans leur PC, se mettent à "faire de la musique".

Lors d'une table ronde tenue après la remise des prix Ars Electronica 2001, Jon Wozencroft, éditeur en chef et directeur artistique de l'étiquette de disques TOUCH, occupait le siège prévu pour Ryoji lkeda, gagnant du Golden Nica dans la catégorie Digital Musics. II réagissait en quelque sorte aux effets pervers de la simplification des processus de création en arts électroniques et souhaitait une musique qui soit davantage préoccupée par la vie plutôt que par ses propres moyens de production lorsqu'il déclara : "With every ten iBook sold, five new glitch $^{2}$ artists and seven labels appear... Cheap technology produces too much crap music. " Wozencroft choisissait tout de même un drôle de lieu pour faire cette sortie ${ }^{4}$, puisque le festival Ars Electronica est connu pour avoir fait une grande place aux artistes de cette nouvelle mouvance. Le jury de l'édition 2000 n'avaitil pas déclaré, s'adressant aux représentants de l' "ancienne influence dominante de la compétition", que ces derniers se devaient, pour prétendre continuer à exister, de "l'éblouir ou de disparaître " ? Et l'introduction de la déclaration du jury de 2001 est, à cet égard, d'une grande clarté :

It's a fact: on the cusp of the twenty-first century, the most innovative, compelling and startling work being produced in the impossibly broad area of Digital Musics comes from musicians whose backgrounds have largely bypassed academic study and customary career paths. Instead their work speaks of an intense, autodidactic engagement with the hyperlinked worlds of post-industrial cultures: conceptual and performance art, installation and video work, improvised music, post-industrial cuttures, eco-activism, postcolonialism, as well as the posttechno/hip hop/dub grassroots diaspora of blunted beatnuts and bedroom boffins.

Cette évolution n'a pas échappé à l'actuel directeur de l'ACREQ, Alain Thibault, qui a au contraire toujours été à l'affût des dernières tendances et qui, bien qu'étant lui-même un compositeur patenté et récipiendaire de plusieurs prix, n'a jamais fait mystère de ses racines "rocks" et de son goût pour les versants les plus bruitistes de cette culture populaire. La direction qu'il a donnée à l'ACREQ depuis le début des années quatre-vingt-dix est parallèle à celle qu'ont empruntée les prix Ars Electronica. On pourrait même dire qu'elle les devance, puisque le récipiendaire du Golden Nica de cette année était l'invité de l'ACREQ l'année dernière, lors de l'édition 2000 de son festival ELEKTRA. Ce festival, qui concentre en quelques jours toute la saison de l'ACREQ, est le seul événement du genre auquel il soit possible d'assister à Montréal, voire en Amérique. On arrive durant ces quelques jours (six cette année, dont trois consacrés au même programme) à couvrir une bonne partie de l'histoire du genre électroacoustique, passant d'un concert acousmatique à un autre du type de ce qu'il est maintenant convenu d'appeler "electronica", donnant un nouveau sens à l'expression "musique mixte" à travers des performances étonnantes. Revoyons en détail la programmation de cette année.
2. Le courant de musique "glitch" travaille surtout à base de résidus sonores électroniques et utilise finalement ce que généralement on laisserait de côté.

3. Cité dans le compte rendu, par Anne Hilde Neset, de la remise des prix Ars Electronica 2001, dans la revue The Wire, $n^{\circ} 212$, octobre 2001, p. 86.

4. Et j'ajouterai que sa propre performance multimédia durant le Festival du nouveau cinéma el des nouveaux médias de Montréal (FCMM), le 18 octobre 2001, n'a pas convaincu beaucoup de monde de ses propres capacités en matière de composition musicale.

5. "In last year's "Forward to the World" jury statement, a call went out to the former dominating influence in the competition to "astonish us or fade away." " Cité dans la déclaration du Digital Music Jury des prix Ars Electronica 2001 : Digital music Diaspora, dans le catalogue Prix Ars Electronica-Cyberarts 2001. 


\section{JOUR 1 - jeudi 8 novembre 2001}

Jean Piché n'est pas le premier venu en matière d'exploration électroacoustique. Études à l'Institut de sonologie d'Utrecht, premier prix en musique électronique au Concours national des jeunes compositeurs canadiens en 1978, premiers enregistrements parus en 1982, développement de logiciels de traitement audionumérique, etc. Il est actuellement directeur du programme d'études électroacoustique et médiatique à l'Université de Montréal et s'intéresse depuis le début des années quatre-vingt-dix au développement de la forme vidéo-musique. Il lui est arrivé de travailler avec des spécialistes de la vidéo, comme l'Américain Tom Sherman, également au programme du festival, mais depuis quelques années il conçoit lui-même les images qu'il nous présente. Dans son œuvre Spin, en version pour trois DVD projetés sur trois écrans vidéo de grandes dimensions et projection sonore multicanaux, c'est l'image qui détermine la composition musicale. Fabriquée "par une technique de tournage en scanning, où la caméra est toujours en mouvement très rapide, menant à une destruction presque complète de l'iconicité du sujet ${ }^{\dagger}$, l'image, extrêmement abstraite, sert en quelque sorte de partition pour la partie musicale. Le résultat est sans doute moins surprenant que ce à quoi Piché nous avait déjà habitué avec son œuvre paNi intiyA, de 1996, où justement, l'iconicité du sujet restait compréhensible et déterminait une musique plus chargée d'émotion. Dans Spin, l'abstraction provoque une langueur qui se traduit en musique par une ambiance méditative créée par des accords soutenus; outre les changements qui surviennent entre les trois mouvements que comporte l'œuvre, le résultat est assez statique, ce qui, après tout, constitue peut-être une réussite pour le compositeur puisque son intention est de créer "une représentation étendue du temps, de la couleur et de la forme musicale ${ }^{7}$.

On nous présentait ensuite la reprise de FausTechnology, œuvre du duo PurForm composé du vidéaste Yan Breuleux et du compositeur Alain Thibault, déjà présentée lors de l'édition précédente du festival. La soirée servait également de lancement de disque pour le duo et l'on trouve sur le disque compact de Purform une séquence Quicktime des dix premières minutes de FausTechnology. Comme pour Spin, la projection utilise toute la surface des trois écrans pour produire une seule image. Ici cependant, l'image projetée est une abstraction géométrique pure, des rectangles iridescents qui respirent au rythme d'une musique d'une simplicité hypnotique et diffusée à une puissance telle que cela procure aux graves une présence physique perceptible par les auditeurs. Le propos de l'œuvre est assez simple : il s'agit d'une transposition du mythe de Faust dans laquelle le rôle du diable est tenu par la "technologie", qui déploie ses charmes afin de nous séduire. Cette dernière aspire littéralement le spectateur-auditeur par une immersion globale produite par "les effets psychosensoriels de la synchronisation du son et de la lumière électronique dans un contexte de diffusion extrême ${ }^{8}$ ". À la
6. Extrait du dossier de presse du Festival ELEKTRA.

7. Ibid.

8. Ibid. 
manière de l'expérience de transe que l'on peut vivre sur un plancher de danse de discothèque, et dont la culture "techno" a presque fait une nouvelle religion, l'œuvre de Purform cherche à hypnotiser le spectateur, mais au contraire de la danse qui peut défouler, l'expérience passive n'apporte rien, sinon un picotement aux yeux. Le développement de l'œuvre, aussi bien visuel que musical, est extrêmement lent et linéaire, ce qui, bien sûr, découle du propos et de l'intention d'hypnotiser le spectateur-auditeur; la dernière partie de l'œuvre ouvre cependant une autre voie, les formes géométriques délaissant les écrans pour être remplacées par des taches noirâtres qui semblent dotées d'une vie balbutiante, tandis que la musique paraît prendre conscience de ses propres pouvoirs de transformation. Comme dans le cas de Jean Piché, il semble que les œuvres précédentes du duo avaient un caractère de surprise, de nouveauté et de trouvaille que $n^{\prime}$ ont pas les $œ u v r e s$ plus récentes. Le grand déploiement du cirque technologique atteint des limites au-delà desquelles il faut arriver à mettre du contenu dans le contenant, sinon il demeure une coquille vide.

\section{JOUR 2 - vendredi 9 novembre 2001}

Le point de vue critique est peut-être plus apte à proposer un "contenu " que l'attitude contemplative, et c'est ce qu'entendait nous démontrer l'artiste multimédia Istvan Kantor, alias Monty Cantsin, par des projections de ses œuvres vidéographiques et surtout par la performance qui allait clôturer cette deuxième soirée intitulée Repossessing the Body-Machine.

Nous avons visionné en première partie trois productions vidéographiques sur lesquelles je ne m'attarderai pas dans le détail, mais dont je dirai tout de même quelques mots. Accumulation est un bombardement $d^{\prime}$ images et de messages pillés, littéralement, dans le flot d'images qui nous submerge chaque jour. Projetées sur trois écrans séparés, les images sont secouées de montages rapides, traversées par des mots et des phrases en forme de slogans apocalyptiques et soutenues par une bande sonore à l'avenant, qui n'explore pas la spatialisation, mais fractionne le temps comme une explosion fragmente l'espace. II s'agit probablement de la plus forte des trois œuvres présentées, mais on a tout de même là trois productions puissantes et c'est leur propre voisinage qui fait de l'ombre aux moins surprenantes. Le traitement rythmique reste à peu près le même dans Broadcast, tandis que Axiom/e est la version vidéo de la performance à laquelle nous assisterons en deuxième partie.

Istvan Kantor, alias Monty Cantsin, est un artiste multidisciplinaire et un provocateur professionnel en révolte contre un monde qu'il considère comme mis à sac par les dictatures médiatiques, politiques et financières. II pratique l'art de la per- 
formance depuis son arrivée en Amérique à la fin des années soixante-dix et a documenté son travail par de nombreux écrits et enregistrements. Influencé tant par le mouvement Dada du début du siècle que par les Situationnistes, il est le fondateur du Néoïsme, un mouvement que je ne tenterai pas de définir ici, lui-même en étant à préparer le sixième recueil de définitions de ce terme... S'il est vrai qu'il a été parmi les premiers durant les années quatre-vingt à enregistrer des disques de chansons sur des musiques électroniques, on ne peut pas dire que l'intérêt premier de sa performance réside dans son élément musical. C'est la critique d'une société envahie par la technologie et pervertie par le cyber-sexe qui, c'est le cas de le dire, retient l'attention. Pendant une bonne heure, Cantsin et ses danseurs et danseuses, transformés en machines humaines animées d'une frénésie sexuelle complètement débridée et évoluant dans le public entre cinq scènes différentes au son d'une musique techno peu inspirée, mais efficace, ont transformé l'Usine $C$ en un temple de la décadence et de la déshumanisation. Amenée au paroxysme par la bande sonore et la projection sur trois écrans d'un nouveau montage du vidéo Axiom/e, mais aussi par la pulsation soutenue d'un DJ officiant à la console, une partie du public se laissera même aller à monter sur la scène centrale pour y manipuler les tiroirs de vieux classeurs de bureau (produisant ainsi des sons qui seront amplifiés). Par-dessus les quatre classeurs installés sur cet autel trônaient deux autres classeurs, couchés sur le dos et dont les tiroirs étaient manipulés par des robots dans une parodie de copulation. Bientôt, les éléments les plus échauffés du public procéderont à la destruction pure et simple des classeurs qu'ils étaient invités à manipuler. Bref, loin d'être un hymne au progrès, la participation de Monty Cantsin à ELEKTRA aura sans doute été le moment le plus agressivement critique de l'incessante prolifération des nouvelles technologies.

\section{JOUR 3 - samedi 10 novembre 2001}

En proposant diverses tendances du genre électroacoustique (pris dans un sens très large), cette troisième soirée du festival est construite sur un modèle qui devrait être étendu plus systématiquement à l'ensemble de l'événement. Le mélange des publics que réunit ce type de programme est sans aucun doute à mettre au crédit du directeur artistique. C'est d'abord Yves Daoust qui ouvrira la soirée avec une suite extraite de son plus récent disque, lancé juste avant le concert avec d'autres productions de l'étiquette Empreintes DIGITALes. Daoust, qui fut l'un des fondateurs de l'ACREQ, en aura sans doute surpris plus d'un en proposant une version vidéomusique de sa pièce Bruits, en trois mouvements, sur des images de Jean-Sébastien Durocher. On est ici dans la plus pure tradition de la musique concrète. Les bruits en question sont ceux de la rue; ceux de la vie diurne dans Children's Corner, ceux de la ville qui dort dans Nuit et ceux de la rumeur estivale dans Fête. Triturés, frag- 
mentés, montés sur huit canaux, les sons renvoient un paysage morcelé, une mosaïque "rendant à la grande nature tout ce qu'ensemble elle avait joint», comme l'écrivait Baudelaire et, dans le cas d'Yves Daoust, le recours à la poésie ne saurait être fortuit. Parce que c'est là, précisément, que réside l'intérêt d'une musique qui ne se laisse pas complètement obnubiler par ses moyens de production.

Venait ensuite le tour de Louis Dufort de nous faire entendre, lui aussi, un extrait de son disque lancé chez Empreintes DIGITALes. Mais, ô surprise, en version acousmatique, c'est-à-dire sans aucun support visuel. Membre du comité artistique de l'ACREQ, Dufort nous présentait sa pièce Décap, qui a reçu une mention dans la catégorie Digital Music lors de la dernière édition des prix Ars Electronica. Soustitrée Étude pour poignets et chevilles, la pièce a été déduite de la musique composée pour un spectacle de la chorégraphe Marie Chouinard et est dédiée "à tous les enfants qui ont été mutilés, violés, assassinés ${ }^{9} \ldots$.. Comme son titre et sa dédicace peuvent le laisser supposer, elle n'est pas de tout repos... Le travail du compositeur sur le matériau de base, essentiellement des voix, dénote une grande maîtrise des techniques propres à l'électroacoustique et nous incite à suivre avec intérêt la suite de la carrière de ce jeune compositeur qui a fait ses premières armes dans la classe d'Yves Daoust au Conservatoire de Montréal.

La suite du programme aurait mieux trouvé sa place dans l'une de ces soirées de "Spoken Words" qui, paraîtil, ont remplacé les soirées de contes de nos grands-pères. Bien sûr, le sujet en était «les nouvelles technologies» et le propos était une critique virulente de la déshumanisation qu'elles provoquent, mais venant après Istvan Kantor, le tout prenait des allures de "Cantsin pour les nuls»... Et le traitement... Tom Sherman monologuant comme un motivateur devant des images (trois, toujours) de personnes filmées dans leur intimité par des caméras reliées à leur ordinateur personnel, pendant que Bernhard Loibner comble les silences de quelques sons puisés dans le sien. A recommander pour un congrès d'informaticiens rongés par la culpabilité.

La fin de cette troisième soirée promettait d'être intéressante, puisque nous allions assister à un concert de Markus Popp, alias Oval, récipiendaire du deuxième prix dans la catégorie Digital Music aux prix Ars Elecronica quelques semaines à peine avant son passage à Montréal. II avait donné une conférence la veille, avant le début des concerts, durant laquelle il nous expliquait son processus de création. II nous a donc exposé très succinctement le fonctionnement d'un logiciel qu'il a créé surtout pour servir d'interface entre ses installations placées dans divers musées ou grandes sociétés à travers le monde, et le public qui les manipule. Un logiciel qui doit être simple à utiliser, donc. II nous a ensuite laissé savoir assez candidement qu'il préférerait que l'histoire se rappelle de lui comme de l'inventeur de ce logiciel, plutôt que pour la musique qui en sort. On a compris ce qu'il voulait dire le lendemain... Si le "fameux" Ovalprocess, qu'il nous a présenté en conférence avec le plaisir d'un enfant se faisant arracher une dent, devait ne consister qu'à empiler les unes par-dessus les autres pendant trop longtemps des
9. Note de programme de la création d'une première version de la pièce durant l'événement Rien à voir 7 , le 8 mars 2000 . 
pistes et des pistes de sons en boucle, comme ce qu'il nous a donné à entendre lors de son concert, je crois pouvoir lui affirmer simplement qu'il n'a rien inventé. Extrait du dossier de presse : I'm doing things live but I'm never sure where it's going. II est trop tentant de dire que ça tourne en rond.

\section{JOUR 6 - samedi 17 novembre 2001}

Deux jours déjà que L'Assemblée, spectacle conçu par Louis-Philippe Demers, fait vibrer la grande salle de I'Usine $C$. Le public est présent en grand nombre pour assister aux évolutions spatiales de 48 "robots", essentiellement des bras mécaniques pourvus chacun d'un haut-parleur et d'un projecteur, fixés à une structure circulaire à l'intérieur de laquelle on peut entrer pour devenir en quelque sorte une bête de foire placée sous observation. On a l'impression d'être en l'an 2000 comme on pouvait se l'imaginer en 1950. On vient assister à un concert de robots. Louis-Philippe Demers fut d'abord éclairagiste et scénographe de haut niveau avant de choisir d'explorer plus à fond la robotique. L'Assemblée est d'abord une installation, c'està-dire qu'elle peut très bien fonctionner toute seule, mais I'ACREQ nous présente en première mondiale la version performance. Le concepteur dirige depuis son ordinateur les robots, les projections et la musique. l'univers dans lequel nous baignons est concentrationnaire au maximum : quatre écrans disposés de part en part de la salle nous renvoient par fragments les images des personnes "enfermées" au centre de la structure, la musique projetée dans la salle rappelle les sirènes d'usine ou d'alerte aérienne et les robots, dans leur chorégraphie, agissent comme les projecteurs d'un mirador cherchant une proie. Ils produisent également des sons stridents par leur propre haut-parleur et leurs mécanismes pneumatiques produisent en direct des sons qui s'ajoutent comme hiératiquement à tous les autres. L'expérience est pour le moins surprenante et a peutêtre plus en commun avec le monde de George Orwell qu'avec celui de Pierre Schaeffer, mais il me semble que cette multiplicité de sources sonores, comme chez Cantsin, ouvre des voies à explorer pour les compositeurs de musique électroacoustique.

En définitive, on serait porté à croire que l'ACREQ fait le bon choix en présentant à la communauté des amateurs de musique de tradition électroacoustique un vaste éventail des diverses recherches en cours, qu'elles émanent du terreau institutionnel ou d'ailleurs. Considérant le nombre de personnes qui s'y sont pressées, le festival ELEKTRA a sans doute constitué un grand succès pour ses organisateurs, et c'est heureux. Le mélange des publics, outre le fait qu'il assure de bonnes entrées aux concerts présentés, peut être tout à fait stimulant pour les créateurs, tandis que la présentation de concerts couvrant des tendances différentes peut 
avoir un effet pédagogique sur le public. Souhaitons seulement que l'ACREQ conserve dans l'avenir le mandat de présenter une vision historique de l'approche électroacoustique, sans oublier ses racines dans la recherche de nouvelles possibilités, puisqu'elle est en définitive le seul organisme à le faire.

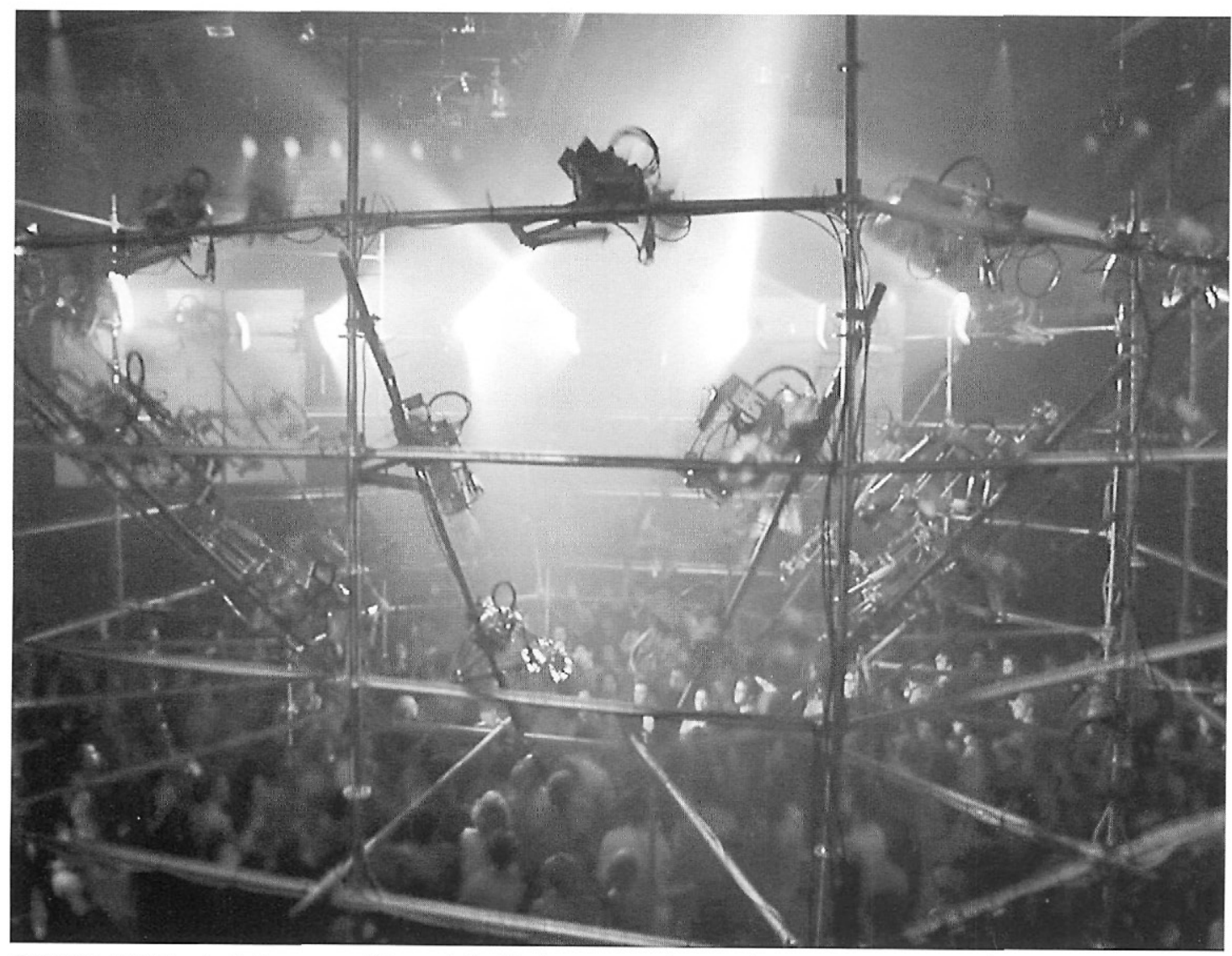

ELEKTRA 2001 - L. P. Demers - L'Assemblée (Performance robotique)

Photo - Peter Dimakos 